\title{
Entendimento dos Médicos Pediatras na Recomendação de Cremes Dentais na Primeira Infância
}

\author{
Understanding of Podiatrists Regarding the Recommendation of Dentifrices in Primary Childhood
}

\section{Danilo Blank ${ }^{1}$}

\section{Abstract}

To characterize the understanding of pediatrician about the recommendation of dentifrices during the first childhood, a broader look is needed attempting not only their effective knowledge, but also the potential knowledge available in the literature. The pediatrician is responsible, at first, for the control of dental caries. Contradictorily, the entity that guides the practices of pediatric primary assistance, i.e. the Brazilian Society of Pediatrics, still does not have a department devoted to oral health. The conduction of systematic reviews suggest the clinician the recommendation of tooth brushing with dentifrice since the eruption of the first tooth, although there is still controversy regarding the use fluoride dentifrice before the age of two years. From a survey conducted by the Brazilian Society of Pediatrics it was observed that $80 \%$ of the pediatricians declared to refer children to a dentist for the first session of preventive care during the first one year of life, which indicates a knowledge - and a pertinent move converging to the recommendations nowadays. The pediatrician and the dentist should establish on of the horizontal connections to provide the children the best available care, which focus and intensity will be based on the permanent evaluation of the risk for caries.

Keywords: Toothpaste; Fluoride.

\section{Resumo}

Para caracterizar o entendimento do pediatra na recomendação de cremes dentais na primeira infância é preciso um olhar mais amplo, que contemple não só seus conhecimentos efetivos, mas também o conhecimento potencial disponibilizado na literatura pediátrica. $O$ pediatra é o responsável primeiro pelo controle da cárie dentária. Contraditoriamente, a entidade que orienta as práticas de assistência primária pediátricas, a Sociedade Brasileira de Pediatria, ainda não tem um departamento devotado à saúde bucal. A realização de revisões sistemáticas sugerem ao clínico recomendar a escovação a partir da erupção do primeiro dente, com escova macia e dentifrício fluoretado, apesar de ainda haver controvérsia sobre o uso deste ultimo antes dos dois anos de vida da criança. A partir de uma enquete da Sociedade Brasileira de Pediatria, observou-se que $80 \%$ dos pediatras alegaram encaminhar as crianças para a primeira consulta com o dentista, para cuidados preventivos, até o final de primeiro ano de vida, o que indica um conhecimento - e uma conduta pertinente - concordando com as recomendações vigentes. $O$ pediatra e o dentista devem estabelecer uma das conexões horizontais para dar à criança o atendimento melhor possível, cujo foco e intensidade se baseará na avaliação permanente do risco de cárie.

Palavras-chave: Dentifrício; Flúor.
${ }^{1}$ Professor associado do Departamento de Pediatria e Puericultura da Faculdade de Medicina da UFRGS; Diretor de Publicações da Sociedade Brasileira de Pediatria

Correspondência: Danilo Blank

Endereço: Rua Ramiro Barcelos 2400, $4^{\circ}$ andar, Porto Alegre-RS, Brasil 90035-003 E-mail: blank@ufrgs.br

\section{Introdução}

Para caracterizar o entendimento do pediatra na recomendação de cremes dentais na primeira infância - usualmente tida como o período que vai do nascimento até o início da idade escolar, aos seis anos - é preciso um olhar mais amplo, que contemple não só seus conhecimentos efetivos (e atitudes correlatas), mas também o conhecimento potencial disponibilizado na literatura pediátrica; algo como um entender de classe.

Em primeiro lugar, porém, vale ressaltar um nó contextual na relação do pediatra com a promoção da saúde bucal, que ainda está por ser equacionado: por um lado, o pediatra tem precedência em todas as questões da atenção primária às crianças - incluindo o controle da cárie -; por outro, a saúde bucal não costuma receber o destaque devido no âmbito das ações médicas de cuidados preventivos.

\section{O pediatra é o responsável primeiro pelo controle da cárie dentária}

A cárie é um problema de saúde pública no Brasil, pois acomete quase um terço das crianças de 18 a 36 meses e $60 \%$ das crianças de cinco anos (BRASIL, 2006). Sua prevenção primária, contudo, não está nas mãos dos odontólogos. Com exceção das grandes intervenções comunitárias, como a fluoretação da água consumida pela população, o trabalho de educação para a saúde bucal se dá primordialmente no âmbito clínico. Estima-se que o número de consultas de lactentes com pediatras seja até 250 vezes maior do que o de consultas com dentistas; além de que muitos pediatras hoje atendem consultas pré-natais, consideradas importantes para a avaliação dos principais riscos de cárie - tais como o nível socioeconômico e educação dos pais - e para a orientação antecipatória acerca dos hábitos que levam à otimização da flora bucal familiar (AMERICAN ACADEMY OF PEDIATRICS, 2008). Logo, os cuidados preventivos da cárie são responsabilidade primária do pediatra e/ou da equipe de saúde que presta atendimento de puericultura, usualmente sob sua coordenação (AMERICAN ACADEMY OF PEDIATRICS, 2011).

\section{Uma negligência histórica e um olhar enviesado}

A prática de puericultura costuma ser balizada por documentos de instituições especializadas, como as "Recomendações para Cuidados de Saúde Pediátricos Preventivos", 
da Academia Americana de Pediatria (AMERICAN ACADEMY OF PEDIATRICS et al., 2007), que tradicionalmente dão pouco relevo para a saúde bucal, há pouco tempo têm recomendado o encaminhamento ao dentista no máximo até o final do primeiro ano de vida e, sobretudo, apoiam-se na avaliação dos fatores de risco para cárie, cuja efetividade tem embasamento questionável (U.S. PREVENTIVE SERVICES TASK FORCE, 2004).

No Brasil, a entidade que orienta as práticas de assistência primária pediátricas, a Sociedade Brasileira de Pediatria, ainda não tem um departamento devotado à saúde bucal - o que se traduz pela ausência completa de qualquer menção a dentifrícios ou prevenção da cárie no seu site (www.sbp.com.br). Como decorrência natural, vários estudos demonstram o desconhecimento dos pediatras acerca das questões de saúde bucal e a falta de orientação adequada das famílias (FREIRE et al., 2000; MALTZ; LACERDA, 2001; BUZALAF et al., 2006; TEIXEIRA et al., 2010). Para além do âmbito do atendimento pediátrico, um estudo recente mostra que os órgãos governamentais brasileiros fornecem recomendações divergentes e não apoiadas em evidências científicas sobre higiene bucal em crianças (SANTOS et al., 2010).

Além disso, prevalece entre os pediatras uma visão anacrônica da cárie, que a aparta das demais doenças infectocontagiosas e menospreza as intervenções preventivas sejam vigentes, como a antissepsia, ou prospectivas, como a imunização ativa - especialmente voltadas ao controle da colonização e transmissão de bactérias patogênicas (MILGROM et al., 2009).

Um prospecto positivo é o site da Academia Americana de Pediatria (www.aap.org), o modelo das sociedades de pediatria do mundo todo, que apresenta uma rica variedade de materiais para profissionais e leigos, incluindo o documento com a política oficial da entidade sobre as intervenções preventivas em saúde bucal a serem empreendidas pelo pediatra (AMERICAN ACADEMY OF PEDIATRICS, 2008) e dois sites acessórios: a Seção de Odontologia Pediátrica (http://www.aap.org/sections/peddentist/default.cfm) e a Iniciativa para a Saúde Oral (http://www.aap.org/commpeds/dochs/oralhealth/). É provável (e desejável) que, no devido tempo, as atividades dos pediatras brasileiros no campo da saúde bucal sigam esse modelo.

\section{Literatura pediátrica: as perspectivas são alentadoras}

Nos últimos anos, a literatura pediátrica tem publicado uma quantidade crescente de estudos de bom padrão científico sobre dentifrícios, ensejando a realização de revisões sistemáticas, que sugerem ao clínico recomendar a escovação a partir da erupção do primeiro dente, com escova macia e dentifrício fluoretado (RAMOSGOMEZ et al., 2007; AMERICAN ACADEMY OF PEDIATRIC DENTISTRY et al., 2009; ROURKE et al., 2010; DAVIES; BRIDGMAN, 2011).

Embora haja controvérsias sobre a segurança da presença de flúor no creme dental nos primeiros dois anos de vida (WALSH et al., 2010) - o que ainda tem levado algumas diretrizes de entidades acadêmicas pediátricas a sugerir que a escovação dental seja feita apenas com água nos dois primeiros anos de vida (CASAMASSIMO; HOLT, 2004; BRASIL, 2006; AMERICAN ACADEMY OF PEDIATRICS, 2009) -, há um quase consenso de que a concentração de flúor do dentifrício seja no mínimo de $1.000 \mathrm{mg} / \mathrm{L}$ até os seis anos de idade (WALSH et al., 2010) e que a redução da quantidade do mesmo na escova seja o melhor modo de manter a eficácia preventiva, reduzindo o risco de fluorose (SCOTTISH INTERCOLLEGIATE GUIDELINE NETWORK, 2005; ELLWOOD; CURY, 2009). Assim, a recomendação da maioria dos especialistas é que quantidade de dentifrício colocada na escova seja um esfregaço nos dois primeiros anos de vida e do tamanho de uma ervilha dos dois aos seis anos (SCOTTISH INTERCOLLEGIATE GUIDELINE NETWORK, 2005; AMERICAN ACADEMY OF PEDIATRICS, 2008; AMERICAN ACADEMY OF PEDIATRIC DENTISTRY et al., 2009).

É interessante citar que a Força Tarefa Estadunidense de Serviços Preventivos, que promove revisões sistemáticas criteriosas para indicar quais procedimentos clínicos têm embasamento científico suficiente para justificar sua inclusão em protocolos de supervisão de saúde, encontrou poucas evidências de que a orientação dada por clínicos ou sua capacidade de realizar triagem de risco resultem em menos cáries e concluiu pela insuficiência de evidências para julgar o equilíbrio entre benefícios e danos da avaliação rotineira de risco na prevenção de doenças dentais em pré-escolares (U.S. PREVENTIVE SERVICES TASK FORCE, 2004). Por outro lado, o Instituto para a Melhora dos Sistemas Clínicos (ICSI), também muito valorizado como base para a formulação de diretrizes pediátricas, incluiu em sua revisão mais recente a orientação sobre higiene bucal - com o uso de dentifrícios fluoretados - entre os serviços clínicos preventivos efetivos (MORRISSEY; PREVENTIVE SERVICES FOR CHILDREN AND ADOLESCENTS WORK GROUP, 2010).

O projeto Bright Futures, a iniciativa mais ambiciosa e abrangente de puericultura científica, vinculada à Academia Americana de Pediatria, incluiu em sua última edição um capítulo inteiro dedicado à promoção da saúde oral. Recomenda a escovação duas vezes por dia a partir da erupção do primeiro dente, mas com a ressalva conservadora de que não se usem dentifrícios fluoretados antes dos dois anos de idade, salvo sob orientação de um dentista, com base no risco elevado de cáries (PROMOTING ORAL HEALTH, 2008).

O Tratado de Pediatria, livro-texto publicado pela Sociedade Brasileira de Pediatria, enfatiza a efetividade do flúor tópico na prevenção da cárie, afirmando que mesmo nos locais onde não há fluoretação da água, os cremes dentais suprem as necessidades diárias para ação tópica, não havendo necessidade de ofertas adicionais. Entretanto, recomenda concentrações de flúor acima de $1.000 \mathrm{mg} / \mathrm{L}$ apenas para crianças maiores de seis anos (NORTON et al., 2010)

Mais recentemente, o livro-texto Nelson Textbook of Pediatrics, virtual bíblia do conhecimento pediátrico, afirma que o uso diário de dentifrício fluoretado deve ser supervisionado, com a recomendação de consenso de uma quantidade do tamanho de uma ervilha para crianças de dois a seis anos, mas ficando também com a ressalva conservadora da Academia Americana de Pediatria de que menores de dois anos utilizem um esfregaço do dentifrício somente se estiverem em risco (sic) de cárie (TINANOFF, 2011).

\section{Entendimentos e atitudes do pediatra brasileiro?}

A Sociedade Brasileira de Pediatria enviou uma enquete por e-mail a cada um dos 15.518 pediatras associados cujo endereço eletrônico tem cadastrado, perguntando: (1) com que idade encaminha as crianças para a primeira consulta com o dentista para cuidados preventivos, (2) se recomenda o uso de dentifrício fluorado para crianças e (3) em caso de resposta positiva, a partir de que idade.

A taxa de respostas foi de apenas $7,2 \%$, considerada muito baixa mesmo para enquetes online. Todavia, a aplicação do modelo 
do continuum de resistência - que assume que os sujeitos que respondem somente depois de um tempo e um esforço consideráveis se assemelham aos não-respondentes quanto aos comportamentos de interesse (KYPRI et al., 2004) -, permite estimar um viés de nãoresposta desprezível, como se vê na tabela 1 , nas quatro colunas à direita dos dados principais, o que valida interpretações baseadas na enquete. Por outro lado, mesmo questionando que a amostra seja representativa dos pediatras brasileiros (por isso o ponto de interrogação no subtítulo acima), as mais de mil respostas dão uma ideia dos entendimentos e atitudes de quem primeiro atende a criança acerca das questões de saúde bucal já referidas.

Como se vê na tabela $1,80 \%$ dos pediatras alegaram encaminhar as crianças para a primeira consulta com o dentista, para cuidados preventivos, até o final de primeiro ano de vida, o que indica um conhecimento - e uma conduta pertinente - concordando com as recomendações vigentes (AMERICAN ACADEMY OF PEDIATRICS, 2008). Mas o fato mais digno de destaque é que, nos dias atuais, a totalidade dos pediatras, pelo menos os respondentes da enquete, encaminha as crianças ao dentista até os três anos de idade.

Tabela 1: Idade de encaminhamento ao dentista

\begin{tabular}{|c|c|c|c|c|c|c|c|}
\hline \multirow{2}{*}{\multicolumn{4}{|c|}{$\begin{array}{c}\text { Com que idade você encaminha as crianças } \\
\text { para a primeira consulta com o dentista } \\
\text { para cuidados preventivos? }\end{array}$}} & \multicolumn{4}{|c|}{$\begin{array}{l}\text { Estimativa do viés } \\
\text { de não-resposta }\end{array}$} \\
\hline & & & & \multicolumn{2}{|c|}{$\begin{array}{c}\text { Primeiros } \\
10 \% \\
\text { a responder }\end{array}$} & \multicolumn{2}{|c|}{$\begin{array}{l}\text { Últimos } 10 \% \\
\text { a responder }\end{array}$} \\
\hline Idade & $\mathrm{n}$ & $\%$ & IC $95 \%$ & $\%$ & IC 95\% & $\%$ & IC $95 \%$ \\
\hline $\begin{array}{l}\text { Antes da erupção } \\
\text { do } 1^{\circ} \text { dente }\end{array}$ & 3 & 0,3 & $0,1-0,7$ & 0 & - & 0 & - \\
\hline $1^{\circ}$ dente & 694 & 57,9 & $55,1-60,6$ & 56 & $\begin{array}{l}46,2- \\
65,3\end{array}$ & 63 & $53,2-71,8$ \\
\hline 1 ano & 276 & 23,0 & $20,7-25,5$ & 21 & $\begin{array}{l}14,7- \\
29,9\end{array}$ & 22 & $15,0-31,1$ \\
\hline 2 anos & 149 & 12,4 & $10,7-14,4$ & 15 & $9,3-23,3$ & 8 & $4,1-15,0$ \\
\hline 3 anos & 77 & 6,4 & $5,2-7,9$ & 8 & $4,1-15,0$ & 7 & $3,4-13,7$ \\
\hline Total & 1199 & 100 & - & 100 & - & 100 & - \\
\hline
\end{tabular}

Quanto ao uso de dentifrícios com flúor, mais de dois terços dos respondentes à enquete afirmaram que não recomendam ou o fazem apenas para crianças com mais de seis anos de idade, o que assinala o desconhecimento das recomendações correntes das autoridades acadêmicas quanto à ênfase que deve ser dada à ação do flúor tópico - em quantidades controladas e supervisionadas - na prevenção da cárie em todas as crianças que tenham dentes (ROURKE et al., 2010; DAVIES; BRIDGMAN, 2011; DYER, 2011).

\section{Conexões horizontais e verticais para além do domicílio "médico"}

No interesse da promoção da saúde bucal, dentre os vários fatos contemporâneos que têm revolucionado as estratégias de acompanhamento da saúde - tais como o olhar ecológico, a continuidade dos cuidados e a prática de decisões compartilhadas -, os mais relevantes são provavelmente a afirmação da medicina baseada em evidências, incluindo sua difusão pela internet (MOYER; BUTLER, 2004; JUNEAU et al., 2011), e a primazia logística do domicílio "médico" - assim entre aspas, para enfatizar que se trata da atenção à saúde como um todo, incluindo a saúde bucal, ainda que ao médico seja por vezes atribuído o papel central (STRICKLAND et al., 2011). O domicílio "médico", que então idealmente assimila o chamado domicílio dentário (AMERICAN ACADEMY OF PEDIATRICS, 2008), é um local definido de referência, de acesso fácil e com equipe conhecida de profissionais, integrada com os recursos da comunidade, capaz de dar atenção abrangente, constante, afetiva, centrada na família, respeitando a sua cultura (NATIONAL CENTER FOR MEDICAL HOME IMPLEMENTATION, 2011).

Tabela 2: Recomendação do uso de dentifrício fluorado

Você recomenda o uso de dentifrício fluorado para crianças? Em caso positivo, a partir de que idade?

\begin{tabular}{lccc}
\hline Idade & $\mathrm{n}$ & $\%$ & IC 95\% \\
\hline $\begin{array}{l}\text { Não } \\
\text { recomendo }\end{array}$ & 452 & 38,0 & $35,3-40,8$ \\
\hline $1^{\circ}$ dente & 75 & 6,3 & $5,1-7,8$ \\
\hline 2 anos & 245 & 20,6 & $18,4-23,0$ \\
\hline 6 anos & 418 & 35,1 & $32,5-37,9$ \\
\hline Total & 1190 & 100 & - \\
\hline
\end{tabular}

Nesse contexto, o pediatra e o dentista podem estabelecer uma das conexões horizontais, para dar à criança o atendimento melhor possível, cujo foco e intensidade se baseará na avaliação permanente do risco de cárie. A tabela 3 mostra uma adaptação da recomendação da Academia Americana de Odontologia Pediátrica, endossada pela Academia Americana de Pediatria (AMERICAN ACADEMY OF PEDIATRIC DENTISTRY, 2010). De acordo com a avaliação do risco, a criança percorrerá mais (e mais cedo) o caminho da referida conexão, com cuidados mais ou menos intensivos quanto à orientação da escovação, aplicação tópica de outros produtos com flúor e monitorização clínica das eventuais cáries. Mas é importante lembrar que o domicílio médico/dentário só funciona se houver uma integração efetiva com a conexão vertical - todos os profissionais e pessoal auxiliar do serviço de saúde - e com a outra conexão horizontal - os programas comunitários de creches, escolas, associações de bairro, igrejas e serviços de saúde pública (HOEKELMAN, 2005; HAGAN et al., 2008). Nesse modelo, cabe aos integrantes mais capacitados de qualquer um dos eixos zelar pelo domínio do programa de promoção da saúde por todos os profissionais envolvidos, pela uniformidade dos padrões de evidência científica e pelo uso racional dos recursos humanos e materiais (BLANK, 2003). 
Tabela 3: Avaliação do risco de cárie - Nascimento até 3 anos (Para médicos e profissionais de saúde não-dentistas)

\begin{tabular}{|c|c|c|c|}
\hline Fatores de risco e resiliência & $\begin{array}{l}\text { Risco } \\
\text { alto }\end{array}$ & $\begin{array}{l}\text { Risco } \\
\text { médio }\end{array}$ & Proteção \\
\hline \multicolumn{4}{|l|}{ Risco biológico } \\
\hline \multicolumn{4}{|l|}{$\begin{array}{l}\text { Pais / cuidadores com } \\
\text { nível socioeconômico baixo }\end{array}$} \\
\hline \multicolumn{4}{|l|}{$\begin{array}{l}\text { Família com padrão } \\
\text { educacional/ } \\
\text { cultural desfavorável }\end{array}$} \\
\hline \multicolumn{4}{|l|}{$\begin{array}{l}\text { Mãe / cuidador com c } \\
\text { áries ativas }\end{array}$} \\
\hline \multicolumn{4}{|l|}{$\begin{array}{l}\text { Criança com >3 refeições/ } \\
\text { bebidas com açúcar por dia }\end{array}$} \\
\hline \multicolumn{4}{|l|}{$\begin{array}{l}\text { Criança colocada na cama } \\
\text { com mamadeira com açúcar }\end{array}$} \\
\hline \multicolumn{4}{|l|}{ Criança com cuidados especiais } \\
\hline \multicolumn{4}{|l|}{ Fatores protetores } \\
\hline \multicolumn{4}{|l|}{$\begin{array}{l}\text { Água fluoretada ou suplemento } \\
\text { de flúor }\end{array}$} \\
\hline \multicolumn{4}{|l|}{$\begin{array}{l}\text { Escovação com dentifrício } \\
\text { fluoretado } 2 x / \text { dia }\end{array}$} \\
\hline \multicolumn{4}{|l|}{$\begin{array}{l}\text { Aplicação tópica de flúor } \\
\text { por profissional }\end{array}$} \\
\hline \multicolumn{4}{|l|}{$\begin{array}{l}\text { Domicílio dentário ou cuidados } \\
\text { dentários regulares }\end{array}$} \\
\hline \multicolumn{4}{|l|}{$\begin{array}{l}\text { Risco indicado por achados } \\
\text { clínicos }\end{array}$} \\
\hline \multicolumn{4}{|l|}{$\begin{array}{l}\text { Criança com manchas brancas } \\
\text { nos dentes }\end{array}$} \\
\hline \multicolumn{4}{|l|}{ Criança com cavidades visíveis } \\
\hline \multicolumn{4}{|l|}{ Criança com placas nos dentes } \\
\hline Avaliação geral do risco de cárie: & & & \\
\hline
\end{tabular}

\section{Referências}

AMERICAN ACADEMY OF PEDIATRIC DENTISTRY; CLINICAL AFFAIRS COMMITTEE; INFANT ORAL HEALTH SUBCOMMITTEE. Guideline on infant oral health care. AAPD Reference Manual, 2009. Disponível em:

$<$ http://www.aapd.org/media/Policies_Guidelines/G_InfantOralHealthC are.pdf $>$

AMERICAN ACADEMY OF PEDIATRIC DENTISTRY; COUNCIL ON CLINICAL AFFAIRS. Guideline on caries-risk assessment and management for infants, children, and adolescents. AAPD Reference Manual, 2010. Disponível em:

<http://www.aapd.org/media/Policies_Guidelines/G_CariesRiskAssess ment.pdf $>$.

AMERICAN ACADEMY OF PEDIATRICS. A pediatric guide to children's oral health. Elk Grove Village, IL: American Academy of Pediatrics, 2009.

AMERICAN ACADEMY OF PEDIATRICS; COMMITTEE ON PEDIATRIC WORKFORCE. Pediatric primary health care. Pediatrics, v. 127, n. 2, p. 397, February, 2011. Disponível em: <http://pediatrics.aappublications.org/content/127/2/397.short>.

AMERICAN ACADEMY OF PEDIATRICS; COMMITTEE ON PRACTICE AND AMBULATORY MEDICINE; BRIGHT FUTURES STEERING COMMITTEE. Recommendations for preventive pediatric health care. Pediatrics, v. 120, n. 6, p. 1376, Dec. 2007. Disponível em: <http://pediatrics.aappublications.org/content/120/6/1376.short >.

AMERICAN ACADEMY OF PEDIATRICS; SECTION ON PEDIATRIC DENTISTRY AND ORAL HEALTH. Preventive oral health intervention for pediatricians. Pediatrics, v. 122, n. 6, p. 1387-1394, Dec. 2008. Disponível em: <http://pediatrics.aappublications.org/content/122/6/1387.abstract >.

BLANK, D. A puericultura hoje: Um enfoque apoiado em evidências. Jornal de Pediatria, v. 79, n. Supl 1, p. S13-22, 2003. Disponível em: < http://www.scielo.br/pdf/jped/v79s1/v79s1a03.pdf >.

BRASIL. MINISTÉRIO DA SAÚDE. SECRETARIA DE ATENÇÃO À SAÚDE. DEPARTAMENTO DE ATENÇÃO BÁSICA. Saúde bucal. Brasília: Ministério da Saúde, 2006. Disponível em: <http://dab.saude.gov.br/docs/publicacoes/cadernos_ab/abcad17.pdf $>$.

BUZALAF, M. A. R. et al. Conhecimento dos médicos pediatras e odontopediatras de bauru e marília a respeito de flúor. Ciência \& Saúde Coletiva, v. 11, p. 201-209, 2006. Disponível em: <http://www.scielosp.org/scielo.php?script=sci_arttext\&pid=S1413$81232006000100029 \&$ nrm=iso $>$.

CASAMASSIMO, P. S.; HOLT, K. A. Bright futures in practice: Oral health pocket guide. Washington, DC: National Maternal and Child Oral Health Resource Center, 2004.

DAVIES, G.; BRIDGMAN, C. Improving oral health among schoolchildren - which approach is best? British Dental Journal, v. 210, n. 2, p. 59-61, 2011. Disponível em: <http://dx.doi.org/10.1038/sj.bdj.2011.1>.

DYER, T. Review: Increasing fluoride concentrations in toothpastes improved prevention of dental caries. Archives of Disease in Childhood Education and Practice Edition, v. 96, n. 4, p. 159, August 1, 2011 2011. Disponível em:

<http://ep.bmj.com/content/96/4/159.short >.

ELLWOOD, R. P.; CURY, J. How much toothpaste should a child under the age of 6 years use? European Archives of Paediatric Dentistry, v. 10, n. 3, p. 168-174, 2009. Disponível em: <http://journal.eapd.gr/2009v10/lssue_3/Vol_10_3_Sept_Ellwood.pdf.

FREIRE, M. C. M.; MACEDO, R. A.; SILVA, W. H. Conhecimentos, atitudes e práticas dos médicos pediatras em relação à saúde bucal. Pesquisa Odontológica Brasileira, v. 14, p. 39-45, 2000. Disponível em:

<http://www.scielo.br/scielo.php?script=sci_arttext\&pid=S151774912000000100008\&nrm=iso >.

HAGAN, J. F. et al. Bright futures: Guidelines for health supervision of infants, children and adolescents. Elk Grove Village, IL: American Academy of Pediatrics, 2008. Disponível em: $<$ http://brightfutures.aap.org/3rd_Edition_Guidelines_and_Pocket_Gui de.html >

HOEKELMAN, R. Commentary: Pre-primary care pediatrics. Pediatrics, v. 115, n. Supplement 3, p. 1148-1149, Apr., 2005. Disponível em:

<http://pediatrics.aappublications.org/content/115/Supplement_3/1148 short $>$ 
JUNEAU, C. E. et al. Evidence-based health promotion: An emerging field. Global Health Promotion, v. 18, n. 1, p. 79-89, 2011.

Disponível em: <http://ped.sagepub.com/content/18/1/79.abstract >.

KYPRI, K.; STEPHENSON, S.; LANGLEY, J. Assessment of nonresponse bias in an internet survey of alcohol use. Alcoholism: Clinical and Experimental Research, v. 28, n. 4, p. 630-4, 2004.

MALTZ, M.; LACERDA, P. Conhecimento do pediatra na área da saúde bucal. Rev ABO Nac, v. 9, n. 4, p. 210-216, 2001.

MILGROM, P.; ZERO, D. T.; TANZER, J. M. An examination of the advances in science and technology of prevention of tooth decay in young children since the surgeon general's report on oral health.

Academic Pediatrics, v. 9, n. 6, p. 404-409, 2009. Disponível em: $<$ http://www.scopus.com/inward/record.url?eid=2-s2.070450277634\&partnerID=40\&md5=70b0a955644e19ad9e78d6d0f20a b6c1 >.

MORRISSEY, L.; PREVENTIVE SERVICES FOR CHILDREN AND ADOLESCENTS WORK GROUP. Preventive services for children and adolescents. 16. Bloomington, MN: Institute for Clinical Systems Improvement, 2010. Disponível em:

<http://www.icsi.org/preventive_services_for_children_guideline_/pre ventive_services_for_children_and_adolescents_2531.html >.

MOYER, V. A.; BUTLER, M. Gaps in the evidence for well-child care: A challenge to our profession. Pediatrics, v. 114, n. 6, p. 1511-1521, Dec. 2004. Disponível em:

<http://pediatrics.aappublications.org/content/114/6/1511.abstract >.

NATIONAL CENTER FOR MEDICAL HOME IMPLEMENTATION [internet]. Elk Grove Village, IL, 2011. Disponível em: <http://www.medicalhomeinfo.org/ >. Acesso em: 23 jun. 2011.

NORTON, R. C. et al. Alimentação do lactente à adolescência. In: LOPEZ, F. A. e JUNIOR, D. C. (Ed.). Tratado de pediatria. Barueri, SP: Manole, 2010. p.1623-1634.

PROMOTING ORAL HEALTH. In: HAGAN, J. F.;SHAW, J. S., et al (Ed.). Bright futures: Guidelines for health supervision of infants, children, and adolescents. 3. Elk Grove Village, IL: American Academy of Pediatrics, 2008. p.155-168.

RAMOS-GOMEZ, F. J. et al. Caries risk assessment appropriate for the age 1 visit (infants and toddlers). Journal of the California Dental Association, v. 35, n. 10, p. 687-702, 2007. Disponível em: $<$ http://www.cda.org/library/cda_member/pubs/journal/jour1007/ramos .pdf $>$.

ROURKE, L. et al. Update on well-baby and well-child care from 0 to 5 years. Canadian Family Physician, v. 56, n. 12, p. 1285-1290,

Dec. 2010. Disponível em:

<http://www.cfp.ca/content/56/12/1285.abstract >.

SANTOS, A. P. P. D.; NADANOVSKY, P.; OLIVEIRA, B. H. D. Survey of brazilian governmental health agencies shows conflicting recommendations concerning oral hygiene practices for children.

Cadernos de Saude Publica, v. 26, p. 1457-1463, 2010. Disponível em: <http://www.scielo.br/scielo.php?script=sci_arttext\&pid=S0102$311 \times 2010000700025 \& n r m=$ iso $>$.

SCOTTISH INTERCOLLEGIATE GUIDELINE NETWORK.

Prevention and management of dental decay in the pre-school child. Edinburgh: Scottish Intercollegiate Guidelines Network 2005.

STRICKLAND, B. B. et al. The medical home: Health care access and impact for children and youth in the united states. Pediatrics, v. 127, n. 4, p. 604-611, Apr. 2011. Disponível em:

<http://pediatrics.aappublications.org/content/127/4/604.abstract >.
TEIXEIRA, A. K. M. et al. Análise dos fatores de risco ou de proteção para fluorose dentária em crianças de 6 a 8 anos em fortaleza, brasil.

Revista Panamericana de Salud Publica, v. 28, p. 421-428, 2010. Disponível em:

<http://www.scielosp.org/scielo.php?script=sci_arttext\&pid=S102049892010001200003\&nrm=iso >

TINANOFF, N. Dental caries. In: KLIEGMAN, R. M.;STANTON, B. F., et al (Ed.). Nelson textbook of pediatrics. 19. Philadelphia, PA: Elsevier Saunders, 2011. cap. 34, p.1254-1256.

U.S. PREVENTIVE SERVICES TASK FORCE. Prevention of dental caries in preschool children, topic page. Apr. 2004. Disponível em: <http://www.uspreventiveservicestaskforce.org/uspstf/uspsdnch.htm>. Acesso: 08 set. 2011.

WALSH, T. et al. Fluoride toothpastes of different concentrations for preventing dental caries in children and adolescents. Cochrane Database of Systematic Reviews, v. 1. 2010. 